Research Article

\title{
The Impact Mechanism and Scenario Simulation of Energy Internet on Transition
}

\author{
Tuo Liu, ${ }^{1}$ Bo Xu, ${ }^{2}$ Xin Zheng, ${ }^{3}$ and Yirui Deng ${ }^{3}{ }^{3}$ \\ ${ }^{1}$ Research Office, State Grid Corporation of China, Beijing, China \\ ${ }^{2}$ State Grid Energy Research Institute Co., Ltd., Beijing, China \\ ${ }^{3}$ School of Economics and Management, China University of Petroleum (East China), Qingdao 266580, China \\ Correspondence should be addressed to Yirui Deng; dengyirui@upc.edu.cn
}

Received 22 February 2021; Revised 29 March 2021; Accepted 9 April 2021; Published 30 April 2021

Academic Editor: Wei Zhang

Copyright (c) 2021 Tuo Liu et al. This is an open access article distributed under the Creative Commons Attribution License, which permits unrestricted use, distribution, and reproduction in any medium, provided the original work is properly cited.

We analyse the direction of the energy revolution from the dimensions of cleanness, electrification, intelligence, and ubiquity. Based on this, we highlight the importance of electricity in the Energy Internet and analyse the challenges faced by the development of the power grid. Then, we propose an electricity-centered energy comprehensive optimization model and set up baseline scenario and carbon neutral scenario, to achieve a systematic simulation of the path of Energy Internet to boost energy transition. The results show that the Energy Internet accelerates the process of clean energy supply, effectively promotes the development of the energy transition, and contributes to the realization of the carbon neutral goal. There are still many problems in the development of the Energy Internet. In order to accelerate the transformation of the energy system and the power industry, it is necessary to establish a sound energy policy system, encourage clean energy consumption, and use the construction of the Energy Internet to achieve optimal allocation of resources.

\section{Introduction}

Energy is an important productivity foundation that supports economic growth and social development, and rapid economic growth is often accompanied by serious problems, such as environmental pollution [1] and resource shortages [2]. Since the twenty-first century, people's requirements for the environment have increased [3] and, a new round of energy revolution marked by the large-scale development and utilization of new energy has been launched worldwide [4]. This round of energy revolution has four directions. The first direction is clean, that is, the increase in the proportion of nonfossil energy in primary energy consumption, the development trend of low-carbon energy instead of high-carbon energy, and zero-carbon energy instead of low-carbon energy. The second is to realize electrification, which means that clean energy generation gradually replaces fossil energy power generation, the proportion of electric energy in terminal energy consumption has increased, and the use of electricity is extended. The third is intelligence, which means to realize the application of a series of advanced technologies such as digital technology and control technology in energy and power systems. The fourth is ubiquity, which means a wide range of optimizing resource allocation and multienergy cosupply, making the scope of electric energy allocation more extensive. In this process, with the application of new technologies and the development of new business and new formats, the trend of network interconnection and mutual benefit among energy subsystems such as electricity, heating, cooling, oil, natural gas, transportation, and so on is increasingly prominent. The concept of "Energy Internet" gradually emerged.

Since 2010, Energy Internet has gradually changed from concept to practical action. In 2011, Jeremy Rifkin, an American science and technology writer, published "The Third Industrial Revolution" [5]. Later, he published the monograph "The Zero Marginal Cost Society" [6], which described the Energy Internet system in which everyone can become an "Energy Prosumers," which has attracted widespread attention and research on the Energy Internet and has begun to heat up. In 2015, at the United Nations 
Development Summit, Chinese President Xi Jinping proposed to build a global Energy Internet and promoted the "China Initiative" to meet global electricity demand in a clean and green way, which received a positive response from the international community. In 2018, the State Grid Corporation of China took the lead in the world to put forward the corporate strategic goal of building an Energy Internet company and carried out a series of active explorations such as source-network-load-storage collaborative interaction, smart energy services (new energy cloud), construction and operation of "SGCC Online," the application of new energy big data in the development of industrial chain, intelligent Internet of vehicles platform, and so on. The construction of China's Energy Internet has thus entered a stage of rapid development.

In 2020, China put forward the vision of "carbon neutrality and carbon peak" at the 75th United Nations General Assembly, in which carbon dioxide emissions strive to reach the peak before 2030 and achieve carbon neutrality by 2060 , and further clarified that the specific targets for 2030 have been set to inject strong impetus into the transition to clean and low-carbon energy. Accelerating the upgrade of the power grid to the Energy Internet is an important direction for China's energy transition to achieve carbon neutrality. On the one hand, the Energy Internet can effectively meet the needs of large-scale grid connection of new energy sources and realize the interconnection, efficient configuration, and comprehensive utilization of various energy sources such as electricity, gas, cold, heat, and hydrogen. On the other hand, it can realize source network load, and the coordination and interaction of storage and storage can effectively support the "plug and play" of various new energy-using facilities. At the same time, it can give birth to new energy business, and new business models and new models facilitate the development of green industries such as energy efficiency services, platform businesses, and the digital economy and promote the entire energy industry system upgrade and evolution. There are three main challenges in upgrading the power grid to the Energy Internet. The first is the challenge of balancing power supply and demand. Wind power and solar power have obvious characteristics of intermittency, volatility, randomness, and antipeak regulation. After large-scale and high-proportion access to the power system, it poses a challenge to the balance adjustment capability of the grid. The second challenge is to keep the system safe and stable. The "weak moment of inertia" of wind power and the "zero moment of inertia" of photovoltaics have led to a significant reduction in the equivalent moment of inertia of the power system and a decline in antidisturbance capability. Distributed energy, electric vehicles, and other "prosumers and consumers" are connected on a large scale, and the system operation characteristics have changed from a one-way flow mode of power flow from the grid to users to two-way interaction, and the complexity of system control has increased significantly. The third is to meet the challenge of upgrading service demand. With the advancement of energy technology and the penetration of Internet technology, user-side needs have become increasingly diversified, personalized, and interactive, which has brought a huge test to the service capability of the power grid. The design standards and operation modes of traditional power grids (mainly distribution networks) cannot meet the above requirements well.

We analyse the challenges faced in the transition from the power grid to the Energy Internet, build an electricitycentered energy comprehensive optimization model, conduct a simulation analysis of the Energy Internet boosting the energy transition, and propose relevant countermeasures and suggestions. The innovation of paper is to electricitycentered energy comprehensive optimization model and conduct a comparative analysis. Not only can it highlight the role of the Energy Internet in the energy transition but it can also identify current challenges and take corresponding measures. This paper is of great significance for exploring the role of the Energy Internet in the energy transition, discovering deficiencies and making corresponding recommendations.

The rest of the paper is organized as follows. Section 2 briefly reviews the related literature on the concept, technology, and application of the Energy Internet. The electricity-centered energy comprehensive optimization model is created in Section 3. In Section 4, on the basis of the model, we carry out the simulation analysis of the Energy Internet boosting the energy transition. Finally, conclusions and suggestions are presented in Section 5; meanwhile, we look forward to the future research content.

\section{Literature Review}

Energy Internet is the product of the integration of energy and Internet, representing the new productivity of the energy system, and has become the focus of attention of domestic and foreign scholars. Jeremy Rifkin believed that characterized by the deep integration of new energy technology and information technology, a new energy utilization system is about to appear, which can be named Energy Internet. Energy Internet is a distributed, open, and shared network based on renewable energy $[5,6]$. The "Guidelines on promoting the development of Internet Plus Smart Energy" proposed by China's National Development and Reform Commission, National Energy Administration, and Ministry of Industry and Information Technology believe that Internet Plus Smart Energy (Energy Internet) is a new form of energy industry development that deeply integrates the Internet and energy production, transmission, storage, consumption, and energy markets. It has the main characteristics of intellectualization of equipment, multifunctional collaboration, information symmetry, decentralized supply and demand, flat system, open transaction, and so on. Global Energy Interconnection Development and Cooperation Organization believes that the Global Energy Internet is based on the UHV power grid as the backbone (channel) and is dominated by the transmission of clean energy, which is a strong smart grid with global interconnection and ubiquity [7]. Zhou Xiaoxin considered that Energy Internet is a networked physical system that directly or indirectly connects the production, transmission, storage, and conversion devices of various primary and secondary energy 
sources, as well as their information communication control and protection devices [8]. State Grid Corporation of China believes that Energy Internet is centered on electricity, based on a strong smart grid, and deeply integrates advanced information and communication technology, control technology, and advanced energy technology to support clean and low-carbon transformation of energy and power, optimize comprehensive energy utilization efficiency, and connect multiple subjects flexibly and conveniently. Energy Internet is a smart energy system with clean, low-carbon, safe and reliable, ubiquitously interconnected, efficiently interactive, and intelligent open feature.

There are four main categories of Energy Internet concepts. The first type emphasizes the expression of the structure of the Energy Internet. This recognition is based on the power grid and uses the Internet to form a new energy network characterized by backbone networks, local area networks, and related connected networks [9, 10]. The second category focuses on the expression of the information Internet, which is the transformation of the existing energy system by the Internet thinking. It is manifested in the free transmission of energy by the open interconnection of multiple energy sources and open peer access [11]. The third type emphasizes the deep combination of Internet technology and energy network to realize the two-way communication between energy and information [12]. The last category emphasizes the combined transmission and optimized use of electricity, heat, and chemical energy. The Internet uses key technologies such as advanced energy storage technology, new energy generation technology, and power electronic technology to realize the close integration of energy networks such as power grids, gas grids, heating power grids, and transportation networks [13-17]. Throughout the many concepts of the Energy Internet, the cores are interlinked, such as source-network-load-storage balance, wide application of digital technology, information communication technology, and control technology, userside participation, co-construction, sharing, open interconnection, and so on. This fully shows that the Energy Internet is actually the result of the superposition of energy technology, digital technology, and Internet thinking.

With the in-depth study of Energy Internet, the application of relevant technologies has also become a research focus. Multiple technologies such as distributed generation, Internet technology, energy storage technology, and intelligent technology have been widely used in practical work $[14,17,18]$. Zhang Xiaoping and others believed that the key factors for the smart grid to switch to the Energy Internet are the development of virtual power plants and demand response technologies and put forward the concept of virtual power systems based on the global power Energy Internet and distribution power energy Internet and compared and analysed virtual power similarities and differences between the system and the virtual power plant [19]. Zha Yabing and others summarized and analysed the 6 key technologies of the Energy Internet: advanced energy storage technology, electronic power transformer technology, intelligent energy management technology, intelligent fault management technology, reliable and secure communication technology, and system planning analysis technology. Related theories have been meaningfully discussed [17]. Yang Fang and others discussed the basic concept of the Energy Internet from the perspective of energy system value and believed that the rapid development of information systems is triggering changes in the way energy system values are created [20]. Based on the analysis of the research status of Energy Internet-related projects at home and abroad, Tian Shiming and others summarized the technical connotation, technical characteristics, technical elements, and technical forms of the Energy Internet and proposed key research technologies for the Energy Internet [14].

In summary, although there have been abundant research contents on Energy Internet, most of them focus on the conceptual framework and technical application of the Energy Internet and less on the impact of Energy Internet on the energy transition. The continuous growth of energy demand has caused the world to face many energy-related challenges, including energy sustainability and global warming. These challenges promote the development and utilization of renewable energy [21]. New energy has advantages, such as being found in large reserves and being clean and renewable, and vigorously developing the new energy industry is an important measure to promote energy transition [22]. Although the promotion of the development and utilization of renewable energy is a common trend in the energy policies of various countries and regions [23], the Energy Internet is based on the future energy system. Under the influence of the multiple goals of the energy development strategy [24-27], the realistic constraints such as cost, risk, resource constraints [28], and the uncertainty [29, 30], the impact of the Energy Internet is different. Therefore, we propose an electricity-centered energy comprehensive optimization model to simulate and analyse the Energy Internet to promote the energy transition and explore the impact of the Energy Internet on the energy transition.

\section{Electricity-Centered Energy Comprehensive Optimization Model}

Energy Internet covers multiple energy resource systems such as coal, oil, natural gas, electricity, and heat. We build an electricity-centered energy comprehensive optimization model to realize the coordination and complementation of multiple energy sources in energy development, transmission, conversion, and comprehensive utilization so as to optimize the energy structure and promote the clean and low-carbon transformation of the energy system. We decompose the problem of comprehensive energy allocation optimization into two modules, which are energy system planning and power system planning. The two modules are gradually refined from top to bottom. The overall framework of the model is shown in Figure 1.

The energy system planning module can simulate the whole process of various energy supply, processing, conversion, and terminal utilization. Among them, the energy supply segment covers coal, oil, natural gas, power, and other industries, while the terminal energy utilization segment covers transportation, business, and other subdivided 


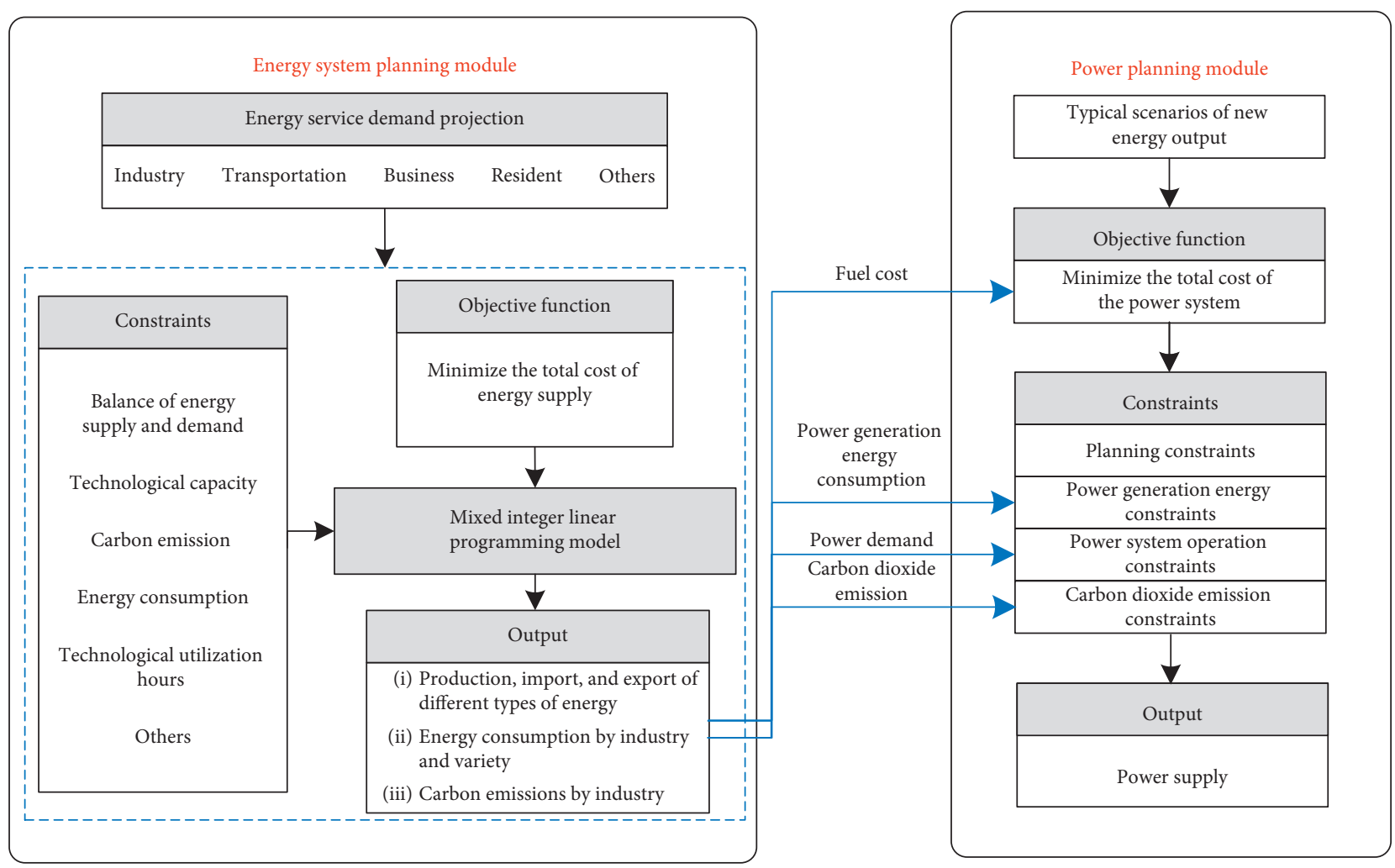

FIGURE 1: Framework of the electricity-centered energy comprehensive optimization model.

industries [31]. Firstly, the energy service demand of various industries can be forecasted by regression analysis, trend extrapolation model, and other methods. The influence factors are economic development, industrial structure, urbanization rate, population, and so on. Based on that the terminal energy demand is obtained by optimizing the terminal energy consumption technology. Then, with the goal of minimizing the total cost of energy supply, considering the national carbon emission target, the development potential of various nonfossil energy sources, and the economics of various energy technologies, various energy resources are optimally allocated to provide boundary conditions of power generation energy consumption, carbon dioxide emissions, and power demand for the power planning module.

The power planning module describes the volatility of large-scale new energy generation in detail through multiple typical scenarios. Under the constraints of the total carbon dioxide emissions of the power system, power generation energy consumption, and system operation, the power supply situation of different future years is optimized to promote the transformation and upgrading of the power grid to the Energy Internet.

3.1. Energy System Planning Module. The optimization goal of energy system planning is to select the technology combination scheme with the minimum total cost of energy supply while meeting the terminal energy demand and various constraints $[32,33]$. We have the following objective function:

$$
\operatorname{Min} \sum_{t=1}^{T} \frac{\operatorname{ANNCOST}(t)-\operatorname{ANNBENiFIT}(t)}{(1+r)^{t-1}}
$$

where $t$ represents the index of the year, $T$ is the planning horizon, and $r$ is the discount rate. $\operatorname{ANNCOST}(t)$ represents cost of the energy system in year $t$, including investment cost, operation and maintenance cost, emission cost, and fuel cost of various industries. ANNBENIFIT $(t)$ is the income of the energy system in year $t$, including equipment residual value.

Energy system planning mainly considers constraints such as balance of energy supply and demand, technological capacity, carbon emission, energy consumption, and technological utilization hours.

3.1.1. Constraint on the Balance of Energy Supply and Demand. In a given year, the local production of coal, oil, natural gas, and other types of energy products plus foreign imports should be balanced by consumption, exports, and changes in inventories.

$$
\begin{gathered}
\text { Ire }_{t, c, \text { imp }}+\sum_{m \in m(c, \text { out })} \text { Flow }_{t, m, c}-\sum_{m \in m(c, \text { in })} \text { Flow }_{t, m, c} \\
-\operatorname{Ire}_{t, c, \exp }-\operatorname{Tra}_{t, c, \text { res }}=0 .
\end{gathered}
$$

In formula (2), $m(c$, out) and $m(c$, in) represent the technology set with output and input of energy product $c$, Flow $_{t, m, c}$ is the input or output of energy product $c$ from technology $m$ in the year $t$, Ire $_{t, c, \exp }$ and Ire $e_{t, c, i m p}$ represent the export and import volume of energy product $c$ in the year 
$t$, respectively, and $\operatorname{Tra}_{t, c, \text { res }}$ is the inventory change of energy product $c$ in the year $t$. For electricity and heat, the annual inventory change is set to 0 .

3.1.2. Technical Capacity Constraint. Various energy extraction, processing, conversion, and terminal utilization technologies need to meet the following formula:

$$
\operatorname{Cap}_{t, m}^{G}=\operatorname{Cap}_{t-1, m}^{G}+\operatorname{Cap}_{t, m}^{N G}-\operatorname{Cap}_{t, m}^{O G} .
$$

In formula (3), $\operatorname{Cap}_{t, m}^{G}$ is the cumulative installed capacity of technology $m$ in the year $t, \operatorname{Cap}_{t, m}^{N G}$ represents the newly installed capacity of technology $m$ in the year $t$, and $\mathrm{Cap}_{t, m}^{O G}$ is the decommissioning capacity of technology $m$ in year $t$.

For a given technology, such as new energy generation and CCUS (carbon capture, utilization, and storage) technology, the following constraint is introduced to guide the development level:

$$
\operatorname{Cap}_{t, m}^{G} \geq \operatorname{Cap}_{t, \text { target}}^{G}
$$

where Cap $\mathrm{C}_{t \text {,target }}$ represents the development goal of capacity of technology $m$ in year $t$.

3.1.3. Carbon Dioxide Emission Constraint. According to the China's proposed carbon dioxide emissions to peak by 2030 and achieve carbon neutrality by 2060, the constraint of country's medium- and long-term carbon dioxide emission levels is defined and the country's carbon dioxide emission constraint is decomposed into subsectors to obtain the upper limit of carbon dioxide emissions for each industry.

$$
\mathrm{EM}_{t, p s}+\sum_{x \in N p s} \mathrm{EM}_{t, x} \leq \mathrm{EM}_{t, \max } .
$$

In formula (5), $\mathrm{EM}_{t, \mathrm{ps}}$ represents the carbon dioxide emissions of the power system in the year $t, N p s$ represents a collection of nonpower industries such as industry, commerce, transportation, and residents, $\mathrm{EM}_{t, x}$ represents the carbon dioxide emissions of industry $x$ in the year $t$, and $\mathrm{EM}_{t, \max }$ represents the national carbon dioxide emission limit in the year $t$.

3.1.4. Energy Consumption Constraint. According to the national energy consumption control policy constraints, the total energy consumption of subsectors is limited.

$$
\mathrm{EC}_{t, p s, c}+\sum_{x \in N p s} \mathrm{EC}_{t, x, c} \leq \mathrm{EC}_{t, c, \text { max}}
$$

where $\mathrm{EC}_{t, p s, c}$ represents the consumption of energy product $c$ in the power system in year $t, \mathrm{EC}_{t, x, c}$ is the consumption of energy product $c$ in industry $x$ in year $t$, and $\mathrm{EC}_{t, c, \max }$ is the upper limit of consumption of energy product $c$ in the year $t$.

3.1.5. Technological Utilization Hours Constraint. In order to ensure the technical economy, the restriction of utilization hours is introduced.

$$
\begin{aligned}
& \operatorname{Act}_{t, m} \geq \frac{U_{t, m}}{8760} \cdot \operatorname{Cap}_{t, m}^{G} \cdot \operatorname{CAPACT}_{m}, \\
& \operatorname{Act}_{t, m}=A_{t, m} \cdot \operatorname{Cap}_{t, m}^{G} \cdot \mathrm{CAPACT}_{m},
\end{aligned}
$$

where Act $_{t, m}$ represents the activity level of technology $m$ in year $t, A_{t, m}$ represents the average availability of technology $m$ in year $t, U_{t, m}$ represents the minimum utilization hour of technology $m$ in year $t$, and $\mathrm{CAPACT}_{m}$ represents the conversion coefficient between the capacity and activity level of technology $m$.

3.2. Power Planning Module. The power planning module is based on the results of the energy system planning, taking into account the various constraints of the power system planning and operation stage for the refined model. In order to take into account the volatility of large-scale new energy output and the seasonal differences between regions, give full play to the complementary characteristics of various power sources across time and space. Based on the historical data of new energy output in various regions, we use a clustering algorithm [34] to obtain typical new energy generation scenarios in each region and use production simulation to characterize power system operating characteristics in different scenarios, with the goal of minimizing system power supply costs.

The power planning optimization goal is as follows:

$$
\operatorname{Min} \sum_{t=1}^{T} \frac{C_{\text {inv }, t}+C_{\text {oper }, t}}{(1+r)^{t-1}}
$$

In formula (8), $C_{\text {inv, } t}$ represents the investment cost of the power system in year $t$ and $C_{\text {oper, } t}$ represents the operating cost of the power system in year $t$.

(1) Power system investment cost is as follows:

$$
C_{\mathrm{inv}, t}=\sum_{z=1}^{Z} \sum_{p \in \Omega} c_{z, t, p}^{\mathrm{inv}} X_{z, t, p}^{\mathrm{inv}}+\sum_{l \in L_{t}} C_{t, l}^{\mathrm{inv} L}
$$

In formula (9), the subscript $z$ represents the region, $Z$ is the total number of regions divided by the system, $p$ is the power supply type, including hydropower, thermal power, and wind power, $\Omega$ represents the power supply type set, $X_{z, t, p}^{\text {inv }}$ indicates the new installed capacity of power $p$ at the region $z$ in year $t, c_{z, t, p}^{\text {inv }}$ represents the investment cost per unit capacity of power supply $p, L_{t}$ represents the set of cross-region transmission channels in year $t$, and $C_{t, l}^{\text {invL }}$ represents the new investment cost of crossregion transmission channel $l$ in year $t$.

(2) System operating cost is as follows:

$$
C_{\text {oper }, t}=\sum_{z=1}^{Z}\left(\mathrm{CM}_{z, t}+\mathrm{CF}_{z, t}+\mathrm{CR}_{z, t}+\mathrm{CC}_{z, t}\right) .
$$


In formula (10), $\mathrm{CM}_{z, t}, \mathrm{CF}_{z, t}, \mathrm{CR}_{z, t}$, and $\mathrm{CC}_{z, t}$, respectively, represent the fixed operating cost, varying operating cost, risk cost, and carbon emission cost at the region $z$ in year $t$.

The fixed operating cost of the system is expressed as follows [35]:

$$
\mathrm{CM}_{z, t}=\sum_{p \in \Omega} c_{z, t, p}^{\text {fixed }} X_{z, t, p}+\sum_{l \in L_{t}} c_{l, t}^{\text {fixed } L} D_{l}^{L} X_{l, t}^{L} .
$$

In formula (11), $c_{z, t, p}^{\text {fixed }}$ is the fixed operating cost per unit capacity of power $p$ at the region $z$ in year $t, c_{l, t}^{\text {fixedL }}$ represents the fixed operating cost of channel $l$ in year $t, X_{z, t, p}$ indicates the cumulative installed capacity of power $p$ at the region $z$ in year $t, X_{l, t}^{L}$ represents the cumulative capacity of transmission channel $l$ in year $t$, and $D_{l}^{L}$ is the length of the crossregion transmission channel $l$.

System varying operating cost is expressed as follows:

$$
\begin{aligned}
\mathrm{CF}_{z, t} & =\sum_{p \in \Omega} X_{z, t, p} H_{z, t, p} \lambda_{z, t, p} f_{z, t, p}, \\
H_{z, t, p} & =\frac{d_{t} \sum_{s \in S} \pi_{z, t, s} \sum_{n=1}^{N} p_{z, t, p, s, n}}{X_{z, t, p}} \\
\sum_{s \in S} \pi_{z, t, s} & =1 .
\end{aligned}
$$

In formula (12), $H_{z, t, p}$ represents the utilization hours of power $p$ at the region $z$ in year $t, d_{t}$ represents the number of days in year $t$, the subscripts $s$ and $n$ represent the new energy generation output scenario and time period, respectively, $S$ is the new energy output scenario set, $N$ represents the number of time periods included in each scenario, $\pi_{z, t, s}$ indicates the probability of occurrence of scenario $s$ at the region $z$ in year $t$, $p_{z, t, p, s, n}$ is the output of power supply $p$ of scenario $s$ and period $n$ at the region $z$ in year $t, \lambda_{z, t, p}$ is the fuel consumption per kilowatt hour of power supply $p$ at the region $z$ in year $t$, and $f_{z, t, p}$ represents the unit cost of fuel $p$ at the region $z$ in year $t$. Considering the impact of local production and cross-region transportation, the average fuel cost is estimated as follows:

$$
f_{z, t, p}=\frac{f_{z, t, p, \mathrm{pro}} F_{z, t, p}^{\mathrm{pro}}+f_{z, t, p, \mathrm{in}} F_{z, t, p}^{\mathrm{in}}+f_{z, t, p, \mathrm{imp}} F_{z, t, p}^{\mathrm{imp}}}{F_{z, t, p}^{\mathrm{pro}}+F_{z, t, p}^{\mathrm{in}}+F_{z, t, p}^{\mathrm{imp}}} .
$$

In formula $(3), F_{z, t, p}^{\mathrm{pro}}, F_{z, t, p}^{\mathrm{in}}$, and $F_{z, t, p}^{\mathrm{imp}}$, respectively, represent the volume of local production, domestic transferred in, and foreign import of fuel $p$ at the region $z$ in year $t$; $f_{z, t, p \text {,pro }}, f_{z, t, p \text {,in }}$, and $f_{z, t, p \text {,imp }}$, respectively, represent the unit production cost, domestic transfer unit cost, and foreign import unit cost of fuel $p$ at the region $z$ in year $t$.

System risk cost is expressed as follows:

$$
\mathrm{CR}_{z, t}=c_{z, t}^{w} R_{z, t}^{w},
$$

where $c_{z, t}^{w}$ represents the penalty cost coefficient of wind and solar power curtailment at the region $z$ in year $t$ and $R_{z, t}^{w}$ is the amount of wind and solar power curtailment at the region $z$ in year $t$.
The system carbon emission cost is expressed as follows:

$$
\mathrm{CC}_{z, t}=\sum_{p \in \Omega} c_{z, t, p}^{\mathrm{carbon}} X_{z, t, p} H_{z, t, p}
$$

where $c_{z, t, p}^{\text {carbon }}$ represents the cost of carbon emission per kilowatt hour of power supply $p$ at the region $z$ in year $t$.

We mainly consider planning constraint, power generation energy consumption constraints, system operation constraints, and carbon emission constraint in the power system planning.

3.2.1. Planning Constraint. In the planning horizon, the energy resource endowment of each region will constrain the upper limit of the installed capacity of power supply, which can be expressed as follows:

$$
X_{z, t, p} \leq X_{z, p}^{\max } .
$$

In formula (16), $X_{z, p}^{\max }$ represents the upper limit of the installed capacity of power supply $p$ at the region $z$.

\subsubsection{Power Generation Energy Consumption Constraints}

(1) Constraint on fossil energy consumption is as follows:

In order to meet the national total energy consumption constraint, the total energy consumption for power generation such as coal and natural gas shall not exceed the upper limit obtained according to the energy system planning module:

$$
\sum_{z=1}^{Z} X_{z, t, p} H_{z, t, p} \lambda_{z, t, p} \leq \mathrm{EC}_{t, p s, p}^{*}
$$

In formula (17), $\mathrm{EC}_{t, p s, p}^{*}$ represents the upper limit of fuel $p$ in year $t$.

(2) Power generation energy balance constraint is as follows [36]:

$$
\begin{aligned}
X_{z, t, p} H_{z, t, p} \lambda_{z, t, p} \leq & F_{z, t, p}^{\mathrm{pro}}+F_{z, t, p}^{\mathrm{in}}-F_{z, t, p}^{\mathrm{out}} \\
& +F_{z, t, p}^{\mathrm{imp}}-F_{z, t, p}^{\exp }+F_{z, t, p}^{\mathrm{res}}-F_{z, t, p}^{\mathrm{Nps}} .
\end{aligned}
$$

In formula (18), $F_{z, t, p}^{\text {out }}, F_{z, t, p}^{\exp }$, and $F_{z, t, p}^{\mathrm{Nps}}$, respectively, represent the domestic transferred out volume, export volume, and nonpower industry consumption of fuel $p$ at the region $z$ in year $t$ and $F_{z, t, p}^{\text {res }}$ represents the initial inventory of fuel $p$ at the region $z$ in year $t$.

The import and export volume of fuel in each region should satisfy the following formula:

$$
\begin{aligned}
& \sum_{z=1}^{Z} F_{z, t, p}^{\mathrm{imp}}=\operatorname{Ire}_{t, p, \mathrm{imp}}^{*}, \\
& \sum_{z=1}^{Z} F_{z, t, p}^{\exp }=\operatorname{Ire}_{t, p, \exp }^{*} .
\end{aligned}
$$


In formula (19), Ire $e_{t, p \text {,imp }}^{*}$ and Ire $e_{t, p \text {,exp }}^{*}$, respectively, represent the national total import and export volume of fuel $p$ in year $t$, which are obtained according to the energy system planning module.

The fuel consumption of nonpower industries should satisfy the following formula:

$$
F_{z, t, p}^{N p s} \leq \sum_{x \in N p s} \mathrm{EC}_{t, x, p}^{*} .
$$

In formula (20), $\mathrm{EC}_{t, x, p}^{*}$ represents the upper limit of fuel consumption in nonpower industries, which is obtained according to the energy system planning module.

The total amount of fuel transferred in and transferred out among various regions in the country should maintain an overall balance.

$$
\sum_{z=1}^{Z} F_{z, t, p}^{\text {in }}=\sum_{z=1}^{Z} F_{z, t, p}^{\text {out }}
$$

\subsubsection{System Operation Constraints}

(1) Power balance constraints are as follows:

$\sum_{p \in \Omega} P_{z, t, p, s, n}+\sum_{l \in L_{t}^{z}} P_{z, t, s, n}^{l}+P_{z, t, s, n}^{\mathrm{dis}}-P_{z, t, s, n}^{\mathrm{cha}}=P_{z, t, s, n}^{\mathrm{load}}$.

In formula (22), $P_{z, t, s, n}^{\text {load }}$ represents the load demand of scenario $s$ period $n$ at the region $z$ in year $t ; P_{z, t, s, n}^{\text {dis }}$ and $P_{z, t, s, n}^{\text {cha }}$, respectively, represent the energy storage discharge and charging power of scenario $s$ period $n$ at the region $z$ in year $t, L_{t}^{z}$ is the set of transmission channels connected to region $z$, and $P_{z, t, s, n}^{l}$ represents the transmission power of the transmission channel $l$ of scenario $s$ period $n$ at the region $z$ in year $t$ and sets the inflow $z$ to the positive direction and the outflow $z$ to the negative direction.

(2) Energy storage charge and discharge constraints [37] are as follows:

$$
\begin{aligned}
P_{z, t, s, n}^{\text {dis }} & \leq g_{z, t, s, n} X_{z, t, \mathrm{sto}}, \\
P_{z, t, s, n}^{\text {cha }} & \leq\left(1-g_{z, t, s, n}\right) X_{z, t, \mathrm{sto}}, \\
\mathrm{SP}_{z, t, s, n} & =\mathrm{SP}_{z, t, s, n-1}+P_{z, t, s, n}^{\text {cha }} \eta_{z, t}^{\text {cha }}-\frac{P_{z, t, s, n}^{\mathrm{dis}}}{\eta_{z, t}^{\text {dis }}}, \\
\mathrm{SP}_{z, t, \min } & \leq \mathrm{SP}_{z, t, s, n} \leq \mathrm{SP}_{z, t, \max } .
\end{aligned}
$$

In formula (23), $X_{z, t \text {,sto }}$ represents the cumulative installed capacity of energy storage at the region $z$ in year $t, g_{z, t, s, n}$ means 0 -1 state variable of storage; when it is 1 , it means the storage is in the state of discharging and when it is 0 , it means the storage is in the state of charging. $\eta_{z, t}^{\text {cha }}$ and $\eta_{z, t}^{\text {dis }}$, respectively, represent energy storage charging and discharging efficiency. $\mathrm{SP}_{z, t, s, n}$ represents the energy stored of scenario $s$ period $n$ at the region $z$ in year $t . \mathrm{SP}_{z, t, \max }$ and $\mathrm{SP}_{z, t, \mathrm{~min}}$, respectively, represent the upper and lower limits of stored energy.

(3) Transmission power constraint of cross-region transmission channels is as follows:

$$
P_{z, t}^{l, \min } \leq P_{z, t, s, n}^{l} \leq P_{z, t}^{l, \max }
$$

In formula (24), $P_{z, t}^{l, m a x}$ and $P_{z, t}^{l, m i n}$ are, respectively, the upper and lower limits of the transmission power of the cross-region transmission channel.

(4) Unit output constraint is as follows.

The output of conventional units such as coal power, natural gas power, and nuclear power in various regions should satisfy the following formula:

$$
P_{z, t, p}^{\min } \leq P_{z, t, p, s, n}^{l} \leq P_{z, t, p}^{\max } .
$$

In formula (25), $P_{z, t, p}^{\min }$ and $P_{z, t, p}^{\max }$, respectively, represent the lower limit and upper limit of output of power supply $p$ at the region $z$ in year $t$.

(5) New energy output constraint is as follows:

$$
\begin{aligned}
& 0 \leq P_{z, t, \text { wind }, s, n} \leq P_{z, t, \text { wind }, s, n}^{*}, \\
& 0 \leq P_{z, t, \text { solar }, s, n} \leq P_{z, t, \text { solar }, s, n}^{*}
\end{aligned}
$$

In formula (26), $P_{z, t, \text { wind }, s, n}^{*}$ and $P_{z, t, \mathrm{solar}, s, n}^{*}$, respectively, represent the theoretical output of wind power and solar power generation of scenario $s$ period $n$ at the region $z$ in year $t$.

(6) Unit climbing rate constraint is as follows:

$$
\begin{gathered}
P_{z, t, p, s, n}-P_{z, t, p, s, n-1} \leq \Delta P_{p}^{\text {up }}, \\
P_{z, t, p, s, n-1}-P_{z, t, p, s, n} \leq \Delta P_{p}^{\text {down }} .
\end{gathered}
$$

In formula (27), $\Delta P_{p}^{\text {up }}$ and $\Delta P_{p}^{\text {down }}$, respectively, represent the upward and downward climbing rate of power supply $p$.

(7) Other constraints are described as follows:

Other constraints mainly include system reserve constraint and curtailment rate constraint.

3.2.4. Carbon Dioxide Emission Constraint. The total carbon dioxide emissions of the power system in each year shall not exceed the upper limit obtained according to the energy system planning module in order to achieve the national carbon dioxide emission reduction target.

$$
\sum_{z=1}^{Z} \sum_{p \in \Omega} X_{z, t, p} H_{z, t, p} \mu_{z, t, p} \leq \mathrm{EM}_{t, p s}^{*} .
$$

In formula (28), $\mu_{z, t, p}$ is the carbon dioxide emission per kilowatt hour of power supply $p$ at the region $z$ in year $t$.

The energy comprehensive optimization model that we constructed is a mixed-integer linear programming model, 
which can be solved with mature optimization software such as GAMS. The medium- and long-term energy transition development path can be optimized by changing the upper limit of carbon emission constraint (equation (5)).

\section{Simulation Analysis}

4.1. Scenario Design. In order to explore the possible path for the Energy Internet to drive China's energy transition in the future, baseline scenario and carbon neutral scenario are designed considering medium- and long-term economic development and growth potential, energy development planning, carbon emission constraint, and other various uncertain factors.

Baseline Scenario. The model uses 2017 as the base year and 2060 as the target year. Assuming that the terminal energy efficiency level rises steadily, electricity is steadily replacing terminal coal, oil, and other fossil energy, and the electrification level is steadily increasing. In 2030, nonfossil energy will account for more than $25 \%$ of primary energy consumption, and the installed capacity of new energy will be expanding. It will reach over 1.2 billion $\mathrm{kW}$ in 2030 and over 3 billion $\mathrm{kW}$ in 2060.

Carbon Neutral Scenario. Considering the acceleration of the energy transition and electrification process, the substantial increase in terminal energy efficiency, and the further acceleration of the development of new energy, various carbon emission reduction technologies such as CCUS will begin to deploy on a large scale after 2030.

\subsection{Simulation Results Analysis}

4.2.1. Primary Energy Consumption. According to the calculation, under the baseline scenario, the total national primary energy consumption in 2025 will be about 5.7 billion tons of standard coal. In the medium and long term, the national primary energy consumption will increase first and then decrease, and it is expected to peak around 2030, with a peak value of about 6 billion tons of standard coal (see Figure 2).

Under the carbon neutral scenario, as the electrification level and energy efficiency level of the whole society are greatly improved, the peak time of total primary energy consumption is earlier than the baseline scenario. The total peak primary energy consumption is about 5.8 billion tons of standard coal, which is about 200 million tons less than that of the baseline scenario. By 2060, the total national primary energy consumption will drop to about 5.1 billion tons of standard coal (see Figure 2).

4.2.2. Carbon Dioxide Emissions. Under the carbon neutral scenario, carbon dioxide emissions from energy consumption are expected to peak between 2025 and 2030, and the peak carbon dioxide emissions are about 10.4 billion tons, which is 600 million tons less than the baseline scenario. It is estimated that by 2060 , with the widespread application of

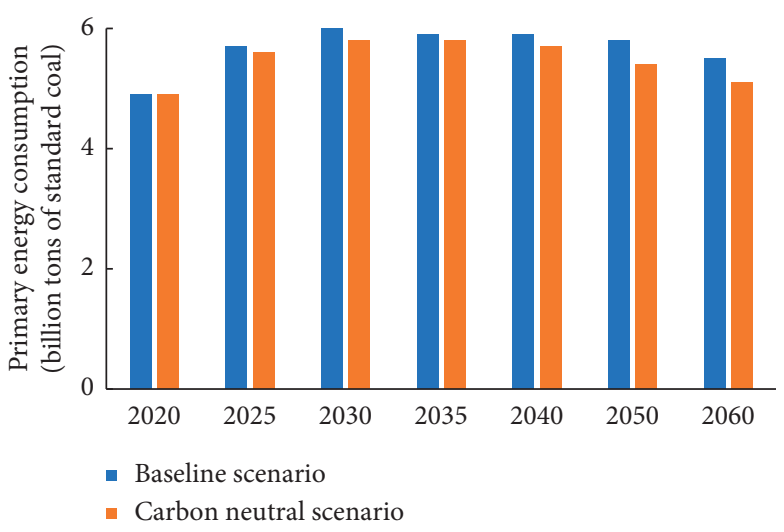

FIGURE 2: National primary energy consumption.

various carbon emission reduction technologies, carbon dioxide emissions will be controlled at about 300 million tons, and this part of carbon dioxide will be absorbed mainly by forest carbon sink, ocean carbon sink, soil carbon sink, and so on (see Figure 3).

The power system is the most important source of carbon dioxide emissions. According to the results predicted by the model, under the carbon neutral scenario, China's power system carbon dioxide emissions will increase from 3.8 billion tons in 2020 to 4.4 billion tons in 2030, accounting for about $44 \%$ of the total carbon dioxide emissions of the energy system. Since then, with the decline in the proportion of coal power and the large-scale application of various carbon emission reduction technologies, the carbon dioxide emissions of the power system have decreased year by year (see Figure 4).

4.2.3. Power Supply. Under the baseline scenario, it is estimated that by 2025,2035 , and 2060, the national installed power capacity will reach 3.1 billion, 4 billion, and 5.4 billion kilowatts, respectively. The installed wind and solar power capacity will surpass coal power around 2035 and become the largest power source (see Figure 5). The proportion of coal power is expected to drop from $49 \%$ in 2020 to $44 \%$, $31 \%$, and $11 \%$ in 2025,2035 , and 2060 , while the proportion of wind and solar power will rise from $24 \%$ in 2020 to $32 \%$, $42 \%$, and $61 \%$, respectively.

Under the carbon neutral scenario, it is estimated that by 2025,2035 , and 2060, the national installed power capacity will reach 3.2 billion, 4.5 billion, and 6.5 billion kilowatts, respectively (see Figure 5). Under this scenario, the peak and decommissioning progress of coal power plants will be further accelerated. It is estimated that by 2060 , the proportion of coal power will drop to about $6 \%$, while the proportion of wind and solar power will increase to about $70 \%$.

The production simulation results of typical scenarios show that with the increase in the penetration rate of new energy, the phenomenon of wind and solar power curtailment began to appear. Take a certain region in the east as an example. Under the baseline scenario, the curtailment rate 


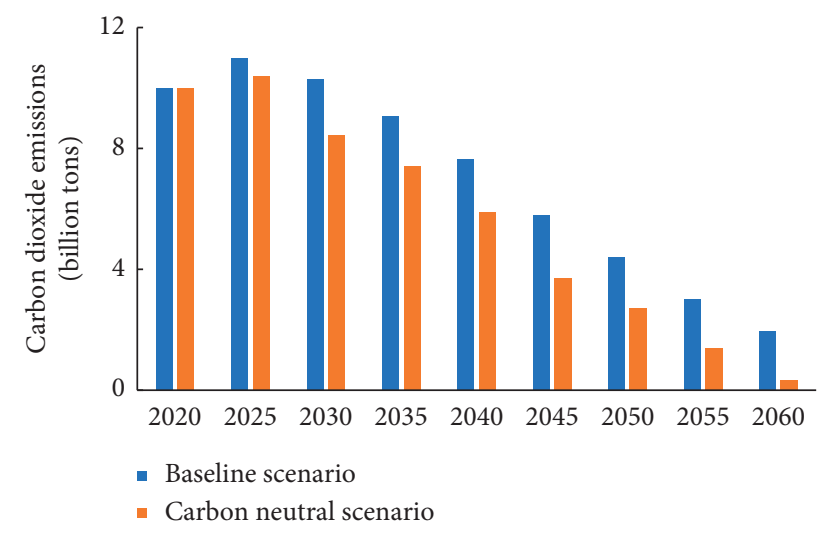

Figure 3: Carbon dioxide emissions from energy systems.

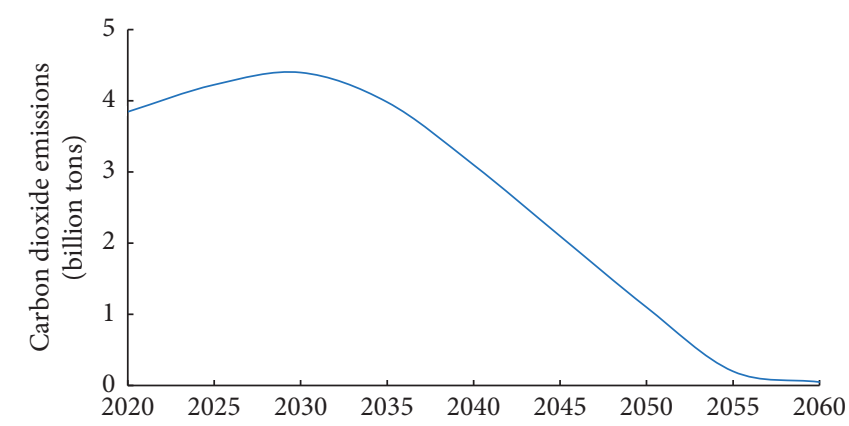

Figure 4: Carbon dioxide emissions from the power system.

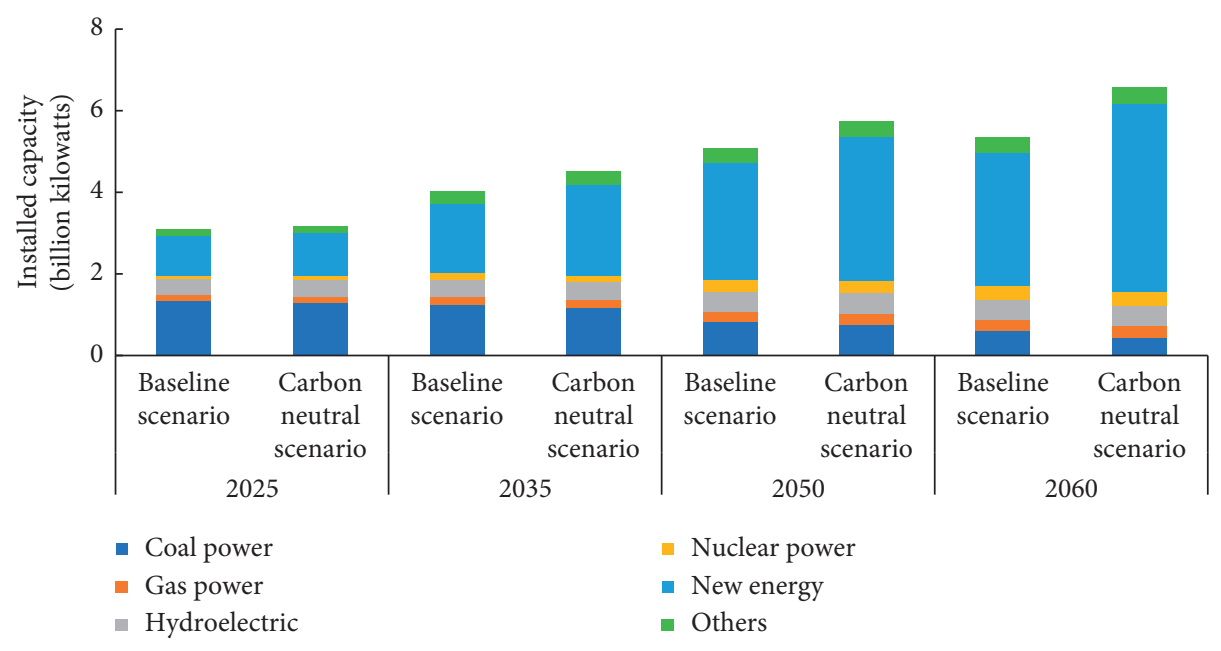

FIGURE 5: Installed capacity of various power sources nationwide from 2025 to 2060.

for the whole year in 2035 is about $0.25 \%$. Under the carbon neutral scenario, as a higher proportion of new energy is connected, the amount of wind and solar power curtailment will increase exponentially. The annual curtailment rate is about $2.2 \%$. It is necessary to coordinate and cooperate with various measures such as the development of energy storage, the improvement of thermal power flexibility, and demand side response to cope with the challenges brought by largescale new energy.

\section{Conclusions}

We construct an electricity-centered energy comprehensive optimization model and conduct simulation analysis of the Energy Internet boosting energy transition by setting different development scenarios. The research results show that with the rapid progress of the energy transition and electrification process and the wide application of various carbon emission reduction technologies, the total primary energy 
consumption is lower than the baseline scenario and the peak time is earlier. The peaking and decommissioning of coal power generation capacity have accelerated, and the proportion of new energy has increased. Carbon emission peak value is reduced compared with the baseline scenario. It can be seen that the Energy Internet can promote clean energy to replace fossil energy, effectively improve the level of electrification of the whole society, reduce carbon emissions, and promote the development of energy transition.

Energy Internet is an important pillar of the third industrial revolution and plays an important role in the energy transition. At present, the construction of the Energy Internet still faces many challenges, such as the high proportion of fossil energy consumption and low energy utilization efficiency. The main suggestions are as follows:

(1) It is necessary to do the top-level design of the Energy Internet in the energy transition, establish a sound energy policy system, and gradually improve the power market. And it is important to building a nationwide power auxiliary service market, set up a reasonable price mechanism and effective incentive model, and guide users to replace fossil energy with clean energy. This is conducive to controlling fossil energy consumption from the source and ensuring that clean energy has sufficient development space.

(2) The construction of the Energy Internet can effectively solve the problem of unreasonable resource allocation. China has unreasonable energy allocation due to the geographical environment and uneven distribution of natural resources. Energy Internet can be fully utilized for energy allocation across time zones and regions to achieve optimal allocation of resources.

(3) The research and development of key technologies such as distributed power generation, Internet technology, energy storage technology, and intelligence technology should be actively carried out to promote the application of Energy Internet technology in the energy transition.

The development of the Energy Internet and the energy transition is very important and urgent. We establish a comprehensive energy optimization model and analyse the role of the Energy Internet in promoting the energy transition. However, there are still many issues that have not been resolved.

Energy Internet and energy transition actually interact. The construction of Energy Internet promotes the further development of energy transition. At the same time, energy transition also provides development opportunities for Energy Internet. In the future, the coupling mechanism between Energy Internet and energy transition can be further studied. And how various factors affect the development of both is also studied.

\section{Data Availability}

The datasets generated and analysed during the current study are available from the corresponding author on reasonable request.

\section{Conflicts of Interest}

The authors declare that they have no conflicts of interest.

\section{References}

[1] W. Zhang, M. Zhang, W. Zhang, O. Zhou, and X. Zhang, "What influences the effectiveness of green logistics policies? A grounded theory analysis," Science of The Total Environment, vol. 714, p. 136731, 2020.

[2] S. Yi and A. Xiao-li, "Application of threshold regression analysis to study the impact of regional technological innovation level on sustainable development," Renewable and Sustainable Energy Reviews, vol. 89, 2018.

[3] C. Wang, Q. Zhang, and W. Zhang, "Corporate social responsibility, Green supply chain management and firm performance: the moderating role of big-data analytics capability," Research in Transportation Business and Management, vol. 37, 2020.

[4] G. Fang, L. Wang, and G. A. O. Zhengye, "Discussion on the coupling mechanism of energy internet and energy transition and identification of influencing factors," Coal Economic Research, vol. 40, no. 473, pp. 13-20, 2020.

[5] S. Ai, "China energy internet business ecological outlook," Software and Integrated Circuit, no. 12, pp. 70-78, 2017.

[6] J. Rifkin, The Zero Marginal Cost Society, CITIC Publishing House, Beijing, China, 2014.

[7] Z. Liu, Global Energy Internet, China Electric Power Press, Beijing, China, 2015.

[8] X. Zhou, S. Chen, Z. Lu, Y. Huang, S. Ma, and Q. Zhao, "Technology features of the new generation power system in China," Proceedings of the CSEE, vol. 38, no. 7, pp. 1893-1904, 2018.

[9] A. Q. Huang, M. L. Crow, G. T. Heydt, J. P. Zheng, and S. J. Dale, "The future renewable electric energy delivery and management (FREEDM) system: the energy," Proceedings of the IEEE, vol. 99, no. 1, pp. 133-148, 2010.

[10] Z. Dong, J. Zhao, F. Wen, and Y. Xue, "From smart grid to energy internet: basic concept and research framework," Automation of Electric Power Systems, vol. 38, no. 15, pp. 1-11, 2014.

[11] H. Sun, Q. Guo, and Z. Pan, "Energy Internet: concept, architecture and Frontier outlook," Automation of Electric Power Systems, vol. 39, no. 19, pp. 1-8, 2015.

[12] M. Zeng, "Energy revolution and energy," China Electrical Equipment Industry, no. 7, pp. 32-34, 2015.

[13] Y. A. N. Taishan, H. Cheng, P. Zeng et al., "System Architecture and key technologies of energy internet," Power System Technology, vol. 40, pp. 105-113, 2016.

[14] S. Tian, W. Luan, D. Zhang, C. Liang, and Y. Sun, "Technical forms and key technologies on energy internet," Proceedings of the CSEE, vol. 35, pp. 3482-3494, 2015.

[15] Y. Yu and C. Qin, "Expatiation on the basic ideas of smart grid," Science China: Information Science, vol. 44, pp. 693-701, 2014.

[16] J. Cao and M. Yang, "Energy Internet towards smart grid 2.0," in Proceedings of the Fourth International Conference on Networking \& Distributed Computing, Los Angeles, CA, USA, December 2013.

[17] Y. Zha, T. Zhang, Z. Huang et al., "Analysis of energy internet key technologies," Science China: Information Science, vol. 44, pp. 702-713, 2014. 
[18] M. Zhao, X. Zhou, and Y. Shang, "Exploring the concept, key technologies and development model of energy internet," Power System Technology, vol. 39, pp. 3014-3022, 2015.

[19] X. Zhang, J. Li, and H. Fu, "Distribution power \& energy Internet: from virtual power plants to virtual power systems," Proceedings of the CSEE, vol. 35, pp. 3532-3540, 2015.

[20] Y. A. N. G. Fang, C. Bai, and Y. Zhang, "Research on the value and implementation framework of energy internet," Proceedings of the CSEE, vol. 35, pp. 3495-3502, 2015.

[21] Q. Feng, X. Sun, J. Hao, and J. Li, "Predictability dynamics of multifactor-influenced installed capacity: a perspective of country clustering," Energy, vol. 214, Article ID 118831, 2021.

[22] S. Yi and Y. Yue-Qi, "Spatial agglomeration of new energy industries on the performance of regional pollution control through spatial econometric analysis," The Science of the Total Environment, vol. 704, Article ID 135261, 2020.

[23] X. Xu, Z. Wei, Q. Ji, C. Wang, and G. Gao, "Global renewable energy development: Influencing factors, trend predictions and countermeasures," Resources Policy, vol. 63, 2019.

[24] X. Xu, Z. Lin, and X. Li, "Multi-objective robust optimization model for MDVRPLS in refined oil distribution," International Journal of Production Research, pp. 1-21, 2021.

[25] X. Xu, C. Wang, and P. Zhou, "GVRP considered oil-gas recovery in refined oil distribution: from an environmental perspective," International Journal of Production Economics, vol. 235, pp. 1-21, 2021.

[26] X. Xu, Z. Lin, and J. Zhu, "DVRP with limited supply and variable neighborhood region in refined oil distribution," Annals of Operations Research, 2020.

[27] X. Xu, J. Hao, and Y. Zheng, "Multi-objective artificial bee colony algorithm for multi-stage resource leveling problem in sharing logistics network," Computers \& Industrial Engineering, vol. 142, Article ID 106338, 2020.

[28] X. Sun, J. Hao, and J. Li, "Multi-objective optimization of crude oil-supply portfolio based on interval prediction data," Annals of Operations Research, 2020.

[29] X. Xu, J. Hao, L. Yu, and Y. Deng, "Fuzzy optimal allocation model for task-resource assignment problem in a collaborative logistics network," IEEE Transactions on Fuzzy systems, vol. 27, no. 5, 2018.

[30] X. Xu, W. Zhang, L. Ning et al., "A bi-level programming model of resource matching for collaborative logistics network in supply uncertainty environment," Journal of the Franklin Institute, vol. 352, no. 9, pp. 3873-3884, 2015.

[31] L. Jia, W. Chen, and D. Liu, "China's low carbon energy development strategy based on China TIMES model system," Qinghua Daxue Xuebao/Journal of Tsinghua University, vol. 51, pp. 525-529, 2011.

[32] G. Fu and S. Wu, "Research on the development mode of clean energy heating and its carbon emission reduction benefit in Beijing," IOP Conference Series: Earth and Environmental Science, vol. 227, Article ID 022025, 2019.

[33] M. Ding and W. Chen, "China's carbon emissions peak pathbased on China TIMES model," Qinghua Daxue Xuebao/ Journal of Tsinghua University, vol. 57, no. 10, pp. 1070-1075, 2017.

[34] Z. Li, C. Wang, J. Liang et al., "Expansion planning method of integrated energy system considering uncertainty of wind power," Power System Technology, vol. 42, pp. 3477-3487, 2018.

[35] Y. Wang, B. Jiao, F. Zhang et al., "Medium and long-term electric power development considering operating characteristics of high proportion of renewable energy," Automation of Electric Power Systems, vol. 41, pp. 9-16, 2017.
[36] J. Bai, S. Xin, J. Liu et al., "Roadmap of realizing the high penetration renewable energy in China," Proceedings of the CSEE, vol. 35, pp. 3699-3705, 2015.

[37] L. Jia, Z. Hu, Y. Song, and H. Ding, "Joint planning of distribution network expansion and distributed energy storage and electric vehicle charging stations," Proceedings of the CSEE, vol. 37, no. 1, pp. 73-84, 2017. 